\title{
Factors predicting survival following complete surgical remission of pulmonary metastasis in osteosarcoma
}

\author{
SAMER SALAH $^{1}$ and SAMAR TOUBASI ${ }^{2}$ \\ ${ }^{1}$ Department of Medical Oncology, King Hussein Cancer Center, Al-Jubeiha 11941; \\ ${ }^{2}$ Department of Clinical Nursing, The University of Jordan, Al-Jubeiha 11942, Amman, Jordan \\ Received July 18, 2014; Accepted September 12, 2014
}

DOI: $10.3892 / \operatorname{mco} .2014 .426$

\begin{abstract}
Pulmonary metastasectomy (PM) is associated with improved survival of patients with metastatic osteosarcoma; however, the factors affecting survival following achievement of complete surgical remission remain controversial. The main objective of this study was to report the outcomes and prognostic factors of osteosarcoma patients who achieved complete remission (CR) following PM. We analyzed the effect of demographic and disease-related characteristics on the overall survival (OS) of consecutive patients with metastatic osteosarcoma who were treated at a single institution and achieved CR following PM, through univariate and multivariate analyses. Between January, 2000 and August, 2013, 62 patients with metastatic osteosarcoma were treated and followed up at our institution. A total of 25 patients achieved CR following PM and were included in this analysis. The 5-year OS and disease-free survival following PM were 30 and $21 \%$, respectively. The factors correlated with inferior OS in the univariate analysis included chondroblastic subtype, post-chemotherapy necrosis $<90 \%$ in the primary tumor, metastasis detected during neoadjuvant or adjuvant chemotherapy and pathological identification of tumor cells reaching the visceral pleural surface of any of the resected nodules. In the multivariate analysis, the chondroblastic subtype was the sole independent adverse prognostic factor $(\mathrm{HR}=4.6,95 \% \mathrm{CI}: 1.0-21.3, \mathrm{P}=0.044)$. Therefore, factors associated with tumor biology, including poor tumor necrosis in the primary tumor and detection of metastasis during primary chemotherapy, are associated with poor post-metastasectomy survival. In addition, chondroblastic subtype and visceral pleural involvement predicted poor prognosis in our series.
\end{abstract}

Correspondence to: Dr Samer Salah, Department of Medical Oncology, King Hussein Cancer Center, Queen Rania Street, Al-Jubeiha 11941, Amman, Jordan

E-mail: ssalah@khcc.jo

Key words: pulmonary metastasectomy, osteosarcoma, complete remission, overall survival, prognosis

\section{Introduction}

Pulmonary metastasectomy (PM) is currently the standard therapy for metastatic osteosarcoma. The evidence supporting the benefit from this surgical intervention is based on retrospective data reporting encouraging 5-year overall survival (OS) rates of 20-40\% and the possibility of cure with this surgical approach. By contrast, death almost always ensues when such patients are treated with systemic chemotherapy alone (1-5).

In the absence of randomized data, retrospective studies have been mainly focused on the analysis of prognostic factors in an attempt to identify selection criteria for the optimal operative candidates (1-5). However, there remains controversy regarding the optimal population that are candidates for PM. Therefore, we performed this retrospective study to investigate factors that may predict long-term survival in our patient population. As PM is aimed at complete resection of the metastases, we aimed to investigate factors associated with survival in a population achieving complete remission (CR) following surgery.

\section{Materials and methods}

Eligible patients and data extraction. Following acquisition of Institutional Review Board approval, we reviewed the medical records of patients with osteosarcoma to identify patients who had undergone PM between January, 2000 and August, 2013 at King Hussein Cancer Center, Amman, Jordan. Patients with gross residual disease following PM (i.e., patients who did not achieve complete surgical remission) were excluded.

The following data were extracted from medical records and filled on data collection sheets: Age at detection of pulmonary metastasis, gender, histological osteosarcoma subtype, mode of pulmonary metastasis (at presentation or at recurrence), timing of pulmonary metastasis (at diagnosis, during neoadjuvant or adjuvant chemotherapy, or following completion of chemotherapy) and the relapse-free interval (RFI), defined as the interval from the resection of the primary tumor until detection of lung metastasis. The RFI was considered to be 0 for patients who presented with primary metastatic disease at initial diagnosis.

In addition, we extracted data on the characteristics of metastatic nodules, therapeutic details, including any perioperative chemotherapy, sites of recurrence and data on repeated PM. 
The common practice at our institution is to initiate multi-agent chemotherapy, including cisplatin and doxorubicin or ifosfamide-containing regimens, prior to PM and to continue such therapy in the adjuvant setting following surgery.

Definitions. We defined resectability as the ability to remove all metastatic nodules without gross residual disease according to the thoracic surgeons' assessment at our institution; this definition also requires a controlled/controllable primary tumor and absence of extrapulmonary metastatic sites at the time of assessment.

We defined CR as absence of any evidence of gross residual disease following $\mathrm{PM}$, irrespective of the status of the microscopic resection margins.

Additionally, we defined OS as the interval from the resection of lung metastasis until the last follow-up or death and disease-free survival (DFS) as the time period following resection of pulmonary metastases during which a patient remained alive with no signs of disease.

Statistical analysis. The Kaplan-Meier survival analysis was applied to assess survival. Demographic and disease-related factors were evaluated with the log-rank test for possible effect on OS; comparisons with $\mathrm{P}<0.05$ were considered to be statistically significant. A multivariate analysis using the backward stepwise Cox regression model was performed on statistically significant variables identified in the univariate analysis. All the statistical analyses were performed using SPSS software, version 16 (SPSS Inc, Chicago, IL, USA).

\section{Results}

Patient characteristics. Between January, 2000 and August, 2013, 135 patients with osteosarcoma of the extremity were treated and followed up at our institution. A total of 62 patients had pulmonary metastasis, either at initial presentation or at recurrence. Of the patients with pulmonary metastasis, 28 underwent PM; 3 of those patients had gross residual disease following surgery and were excluded ( 2 with residual unresectable chest wall and pleural metastasis and 1 due to refusal of a planned above-knee amputation to treat the primary tumor). The 25 patients who were free of gross residual disease following PM were included in this analysis (5 patients with metastasis at diagnosis and 20 patients with metastasis at the time of relapse).

The sites of the primary tumors were the femur in 14 cases, the tibia in 7 cases and the humerous in 4 cases. Data on pathological necrosis of the primary tumor were available for 23 of the patients, of whom 6 had necrosis $\geq 90 \%$ and 17 had necrosis $<90 \%$.

The median RFI for patients who presented with metastasis at relapse was 17.6 months (range, 4.5-63 months).

In the 5 patients with metastasis at diagnosis, the primary tumor and the pulmonary metastases were completely resected in staged procedures following chemotherapy, which was initiated in a neoadjuvant setting and continued as adjuvant therapy following surgery; all the patients underwent resection of the primary tumor prior to PM. In the 20 patients with metastasis at relapse, locoregional recurrences were observed in 4 patients and were completely resected prior to PM. Of the 20 patients with metastasis at relapse, 15 received perioperative chemotherapy.

A total of 14 patients had unilateral pulmonary metastatic disease, whereas 11 underwent resection of bilateral metastatic pulmonary nodules through staged thoracotomies. As regards the number of surgeries, the staged thoracotomy for bilateral pulmonary nodules was considered as one PM surgery.

A total of 10 patients underwent resection of solitary metastatic nodules, 6 patients underwent resection of 2-5 nodules and 7 patients underwent resection of $>5$ nodules (range, 6-21 nodules) during the first PM. A total of 8 patients required repeated PM (range, 2-3 metastasectomies). Apart from 1 patient who required a lobectomy, all the other patients underwent resection of the pulmonary nodules through wedge resections during the first PM. In 5 patients, at least one of the nodules was resected with a positive margin ( $\mathrm{R} 1$ resection) in the first PM. Furthermore, 5 patients had detectable tumor cells reaching the visceral pleural surface in at least one of the resected nodules detected at pathological examination of the resected specimen. There was no reported mortality within 1 month of the first PM.

Recurrence and survival outcomes. Following PM, 8 patients remained free of disease and 17 had documented recurrences at a mean interval of 8.7 months (range, 3-35 months) following the first PM. The first recurrence was limited to the lungs in 11 patients, 8 of whom had repeated PMs, as mentioned above. The remaining 6 patients had lung recurrence in addition to at least one extrapulmonary site as their first presentation of recurrent disease; bone metastasis occurred in 4 of those patients. None of the patients had extrapulmonary metastasis without lung recurrence. The 5-year RFS for the entire cohort was $21 \%$.

The median follow-up for the patients following PM was 25 months (range, 2-62 months). At the time of this analysis, 15 patients had succumbed to the disease, whereas none of the patients had died from other causes. The OS rate was $30 \%$ at 5 years (median survival time, 25 months).

The results of the univariate analysis for the effect of factors of interest on OS are summarized in Table I.

Patients with the chondroblastic subtype exhibited inferior OS compared to patients with other histological subtypes, with a 5-year OS of 13 and 37\%, respectively ( $\mathrm{P}=0.014$; Fig. 1). The degree of pathological necrosis in the primary tumor following neoadjuvant chemotherapy also exerted a significant effect on OS, with a 5-year OS of 8 and $69 \%$ for tumor necrosis of $<90$ and $\geq 90 \%$, respectively ( $\mathrm{P}=0.011$; Fig. 2 ).

Although the RFI did not affect OS, we observed a significant association between the timing of detection of metastasis in relation to chemotherapy and survival. Patients with metastasis detected during neoadjuvant or adjuvant chemotherapy fared significantly worse compared to patients whose metastases were detected at initial diagnosis or following completion of chemotherapy; the 5-year OS rate for the latter group was $33 \%$, whereas patients with metastasis detected during neoadjuvant or adjuvant chemotherapy exhibited a 1-year OS of $0 \%$ ( $\mathrm{P}<0.0001$; Fig. 3).

Based on our analysis, patients with positive resection margins in any of the resected nodules did not have statistically significant differences in OS compared to patients with nega- 
Table I. Results of univariate analysis.

\begin{tabular}{|c|c|c|c|c|}
\hline Factors & $\begin{array}{l}\text { Patient no. }(\%) \\
\qquad(\mathrm{n}=25)\end{array}$ & Median OS (months) & 5-year OS (\%) & Log-rank P-value \\
\hline \multicolumn{5}{|l|}{ Age (years) } \\
\hline$<20$ & $10(40)$ & Unreached & 58 & 0.064 \\
\hline$\geq 20$ & $15(60)$ & 23.2 & 14 & \\
\hline \multicolumn{5}{|l|}{ Gender } \\
\hline Male & $18(72)$ & 25 & 21.6 & 0.35 \\
\hline Female & 7 (28) & 33.3 & 43 & \\
\hline \multicolumn{5}{|l|}{ Histological subtype } \\
\hline Chondroblastic & $8(32)$ & 13.4 & 13 & 0.015 \\
\hline Other & $17(68)$ & 33.3 & 37 & \\
\hline \multicolumn{5}{|l|}{ Tumor necrosis } \\
\hline$<90 \%$ & $17(68)$ & 23.2 & 8 & 0.011 \\
\hline$\geq 90 \%$ & $6(24)$ & Unreached & 69 & \\
\hline ND & $2(8)$ & & & \\
\hline \multicolumn{5}{|l|}{ RFI (months) } \\
\hline$<12$ & $11(44)$ & 24.6 & 30 & 0.74 \\
\hline$\geq 12$ & $14(56)$ & 29 & 27 & \\
\hline \multicolumn{5}{|c|}{ Peri-PM chemotherapy } \\
\hline Yes & $20(80)$ & Unreached & 63 & 0.13 \\
\hline No & $5(20)$ & 24.6 & 19 & \\
\hline \multicolumn{5}{|l|}{ Number of nodules } \\
\hline$<3$ & $12(48)$ & 25 & 27 & 0.85 \\
\hline$\geq 3$ & $13(52)$ & 24.8 & 29 & \\
\hline \multicolumn{5}{|l|}{ Laterality } \\
\hline Unilateral & $13(52)$ & 25 & 33 & 0.58 \\
\hline Bilateral & $12(48)$ & 29 & 26 & \\
\hline \multicolumn{5}{|l|}{ Size (cm) } \\
\hline$<3$ & $11(44)$ & 32 & 36 & 0.91 \\
\hline$\geq 3$ & $11(44)$ & 24 & 30 & \\
\hline ND & $3(12)$ & & & \\
\hline \multicolumn{5}{|c|}{ Metastasis during CTX } \\
\hline Yes & $3(12)$ & 11.0 & 0 & $<0.0001$ \\
\hline No & $22(88)$ & 29.0 & 33 & \\
\hline \multicolumn{5}{|c|}{ Detection of metastasis } \\
\hline At initial diagnosis & $5(20)$ & 32.0 & 40 & 0.43 \\
\hline At recurrence & $20(80)$ & 24.8 & 27 & \\
\hline \multicolumn{5}{|c|}{ Status of resection margins } \\
\hline R0 & $19(76)$ & 25 & 38 & 0.36 \\
\hline $\mathrm{R} 1$ & $6(24)$ & 29 & 0 & \\
\hline \multicolumn{5}{|c|}{ Visceral pleural involvement } \\
\hline Yes & $5(20)$ & 13.1 & 0 & 0.007 \\
\hline No & $20(80)$ & 32 & 39 & \\
\hline
\end{tabular}

OS, overall survival; ND, no data; RFI, relapse-free interval; PM, pulmonary metastasectomy; CTX, chemotherapy.

tive resection margins, although there was a non-significant tendency towards an inferior OS for patients with positive margins. However, the detection of malignant cells at the visceral pleural surface of any of the resected pulmonary nodules was correlated with inferior survival; the 3-year OS rate was $0 \%$ for patients with visceral pleural involvement and the 5-year OS rate was $39 \%$ for patients with no visceral pleural involvement ( $\mathrm{P}=0.007$; Fig. 4).

In the multivariate analysis, the chondroblastic subtype was the sole independent prognostic factor $(\mathrm{P}=0.044)$, whereas the effect of metastasis detected during chemotherapy on survival approached statistical significance $(\mathrm{P}=0.051$; Table II). 


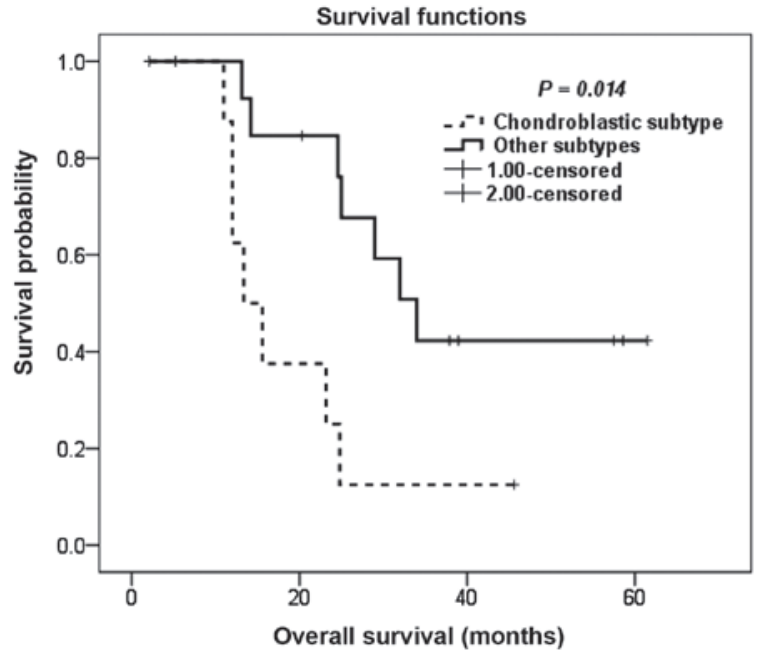

Figure 1. Kaplan-Meier overall survival estimation following pulmonary metastasectomy according to the histological subtype.

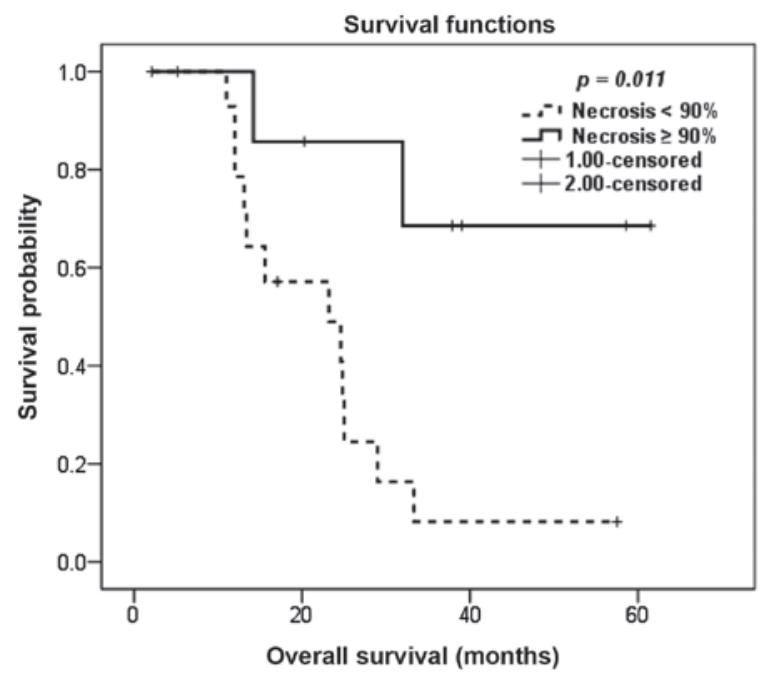

Figure 2. Kaplan-Meier overall survival estimation following pulmonary metastasectomy according to extent of pathological necrosis in the primary tumor.

Finally, we assessed the effect of a number of prognostic factors that we identified in the univariate analysis on OS outcomes and observed significant differences in survival between three groups of patients: The median OS for patients with $0-1$ risk factors, patients with 2 risk factors and patients with $\geq 3$ risk factors was unreached, 23.2 months and 12.0 months, respectively ( $\mathrm{P}<0.0001$; data not shown).

\section{Discussion}

Approximately 30-40\% of patients with initially non-metastatic osteosarcoma eventually develop lung metastasis (6-8) and almost half of these relapses become evident within 18 months of continuation of chemotherapy $(9,10)$. Furthermore, 10-20\% of osteosarcoma patients present with metastatic disease at initial diagnosis $(11,12)$.

An important characteristic of metastasis in osteosarcoma that makes PM an attractive therapeutic option is the propensity

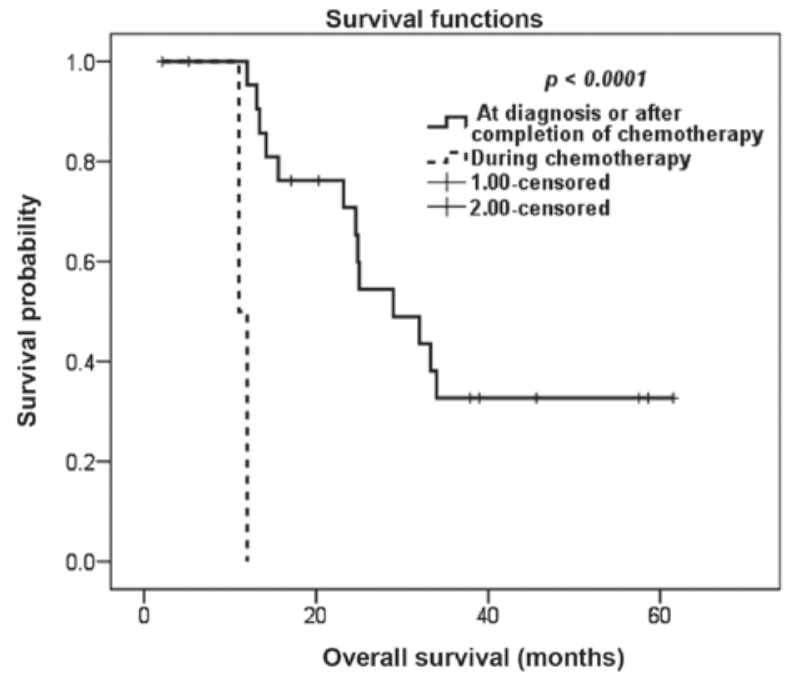

Figure 3. Kaplan-Meier overall survival estimation according to the timing of detection of metastasis in relation to primary chemotherapy.

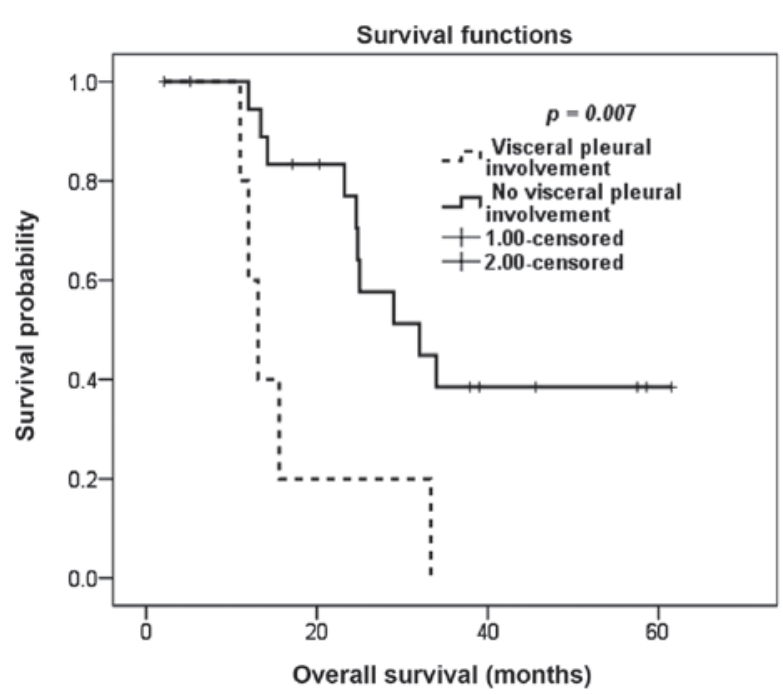

Figure 4. Kaplan-Meier overall survival estimation according to pathological identification of visceral pleural involvement.

for isolated pulmonary metastasis, which is the most common pattern of systemic recurrence, contributing to $65-80 \%$ of relapses $(10,13)$. Furthermore, isolated pulmonary metastasis is the most common pattern of metastasis in patients with primary metastatic disease (12).

The prognostic factors for survival following PM in osteosarcoma are controversial; therefore, the selection of optimal candidates for this type of surgery is a subject under debate. Although the achievement of CR is the most consistently identified prognostic factor $(1,10,12-16)$, contradicting data have been reported regarding the significance of the other factors.

Data from literature have been inconsistent regarding the effect of the number of pulmonary nodules on post-metastasectomy survival. Certain studies suggested that the number of nodules adds prognostic information $(1,5,7,8,17)$, whereas other studies failed to confirm a significant association between the number of nodules and survival $(18,19)$. In our study, we did not identify any prognostic correlation between 
Table II. Results of multivariate analysis for factors affecting overall survival.

\begin{tabular}{lcc}
\hline Clinical variables & Hazard ratio & $95 \%$ CI \\
\hline Histological subtype & 4.6 & $1.0-21.3 .$. \\
Chondroblastic & 1 & 0.044 \\
Other subtypes & & $0.73-20.0$ \\
Extent of primary tumor necrosis & 3.80 & 0.092 \\
$<90 \%$ & 1 & $0.93-126.4$ \\
$\geq 90 \%$ & 10.8 & 0.051 \\
Detection of metastasis & 1 & $0.31-5.26$ \\
During chemotherapy & 1.3 & 0.730. \\
At diagnosis or after completion of chemotherapy & 1 & \\
Visceral pleural involvement & & \\
Present & & \\
Absent &
\end{tabular}

CI, confidence interval.

the number of resected nodules and survival, suggesting that, when all the nodules are completely resected, their number loses its significance as a prognostic factor.

There is evidence in the literature that the timing of pulmonary metastasis is associated with prognosis. Certain studies demonstrated that the time interval from the resection of the primary tumor until the detection of pulmonary metastasis affects survival $(8,19)$, whereas other studies suggested that the timing in relation to chemotherapy is significant in terms of prognosis $(1,2)$. In our study, we did not identify any correlation between the RFI and survival. Furthermore, we did not observe any difference in survival between patients with metastasis at diagnosis and those with metastasis at recurrence; however, patients whose metastases were detected during treatment with neoadjuvant or adjuvant chemotherapy fared significantly worse following PM compared to those whose metastases were detected at initial diagnosis or following completion of chemotherapy. Similar to our data, other studies demonstrated that the timing of metastasis in relation to chemotherapy correlates with survival $(1,2)$.

Progression on adjuvant chemotherapy may be a confounding factor that leads to an apparent correlation between RFI of $<1$ year and OS in certain studies. It may be hypothesized that the subgroup of patients who relapsed within 1 year of definitive therapy of the primary tumor includes a proportion of patients who developed disease progression during adjuvant chemotherapy and, as such, the survival of the entire subgroup may appear worse, as it includes a subpopulation of patients whose disease is refractory to chemotherapy.

Consistent with our data, Harting et al demonstrated a correlation between the degree of necrosis in the primary tumor following neoadjuvant chemotherapy and post-metastasectomy survival (19). It was demonstrated that the extent of necrosis in the primary tumor was also correlated with the survival of patients who did not achieve surgical remission (12). By contrast, certain studies failed to identify any correlation between post-metastasectomy survival and the extent of necrosis in the primary tumor (20) or in the resected pulmonary metastases (7).
There is currently paucity of data in the literature regarding the effect of histological subtype on survival following PM in osteosarcoma, as the majority of the studies do not include this factor in their analysis. To the best of our knowledge, our study is the first to suggest an association between the chondroblastic subtype and survival following PM. In addition, the prognostic effect of pathological identification of visceral pleural involvement in any of the resected nodules on patient survival has not been previously reported for patients with metastatic osteosarcoma. However, until additional confirmation of these prognostic factors in further large-scale studies, the effect of those factors on survival must be interpreted with caution due to the limited number of patients in our series.

The contribution of perioperative chemotherapy to the improved survival of patients with metastatic osteosarcoma who achieved CR following surgery remains unclear. Although there have been data demonstrating that chemotherapy is associated with improved survival in patients with relapsed osteosarcoma who have unresectable metastatic disease, several studies reported that administering chemotherapy does not result in an additional improvement in the survival of patients with metastatic ostrosarcoma who achieve complete surgical remission $(13,15)$. However, a subanalysis on the effect of perioperative chemotherapy on the survival of patients who achieved complete surgical remission demonstrated a survival advantage for the subgroup with $>3$ lung nodules (15). Therefore, some investigators suggest withholding perioperative chemotherapy in patients with disease characteristics suggesting less aggressive tumor biology, such as patients with solitary nodules and prolonged RFI.

The main limitations to our study were the small sample size, rendering any exploratory subanalysis infeasible, the retrospective design and our inability to report quality of life for the included patients. In addition, the respiratory status following PM was not addressed in the present study, which is a consistent limitation among all studies according to a recent systematic review (21). However, despite these limitations, our study may provide additional information on the current outcomes following PM in osteosarcoma, given the fact that 
all of our patients were treated recently and in a setting of multimodality therapy and, as such, may be more relevant to the current clinical practice. In addition, our study suggested that chondroblastic histological subtype and visceral pleural involvement of the resected nodules may add important prognostic information; however, additional confirmation of those two identified prognostic factors is required by future studies.

In conclusion, $\mathrm{PM}$ is associated with long-term survival and the possibility of cure for patients with pulmonary metastatic osteosarcoma. Factors associated with less favorable tumor biology, including poor necrosis in the primary tumor, detection of metastasis during primary chemotherapy and chondroblastic subtype predicted poor prognosis. In addition, pathological identification of visceral pleural involvement in any of the resected pulmonary nodules also predicted poor survival in our series.

\section{References}

1. Chen F, Miyahara R, Bando T, et al: Prognostic factors of pulmonary metastasectomy for osteosarcomas of the extremities. Eur J Cardiothorac Surg 34: 1235-1239, 2008.

2. Huang YM, Hou CH, Hou SM and Yang RS: The metastasectomy and timing of pulmonary metastases on the outcome of osteosarcoma patients. Clin Med Oncol 14: 99-105, 2009.

3. Saltzman DA, Snyder CL, Ferrell KL, Thompson RC and Leonard AS: Aggressive metastasectomy for pulmonic sarcomatous metastases: a follow-up study. Am J Surg 166: 543-547, 1993.

4. Temeck BK, Wexler LH, Steinberg SM, McClure LL, Horowitz $\mathrm{M}$ and Pass HI: Metastasectomy for sarcomatous pediatric histologies: results and prognostic factors. Ann Thorac Surg 59: 1385-1389, 1995.

5. Suzuki M, Iwata T, Ando S, et al: Predictors of long-term survival with pulmonary metastasectomy for osteosarcomas and soft tissue sarcomas. J Cardiovasc Surg (Torino) 47: 603-608, 2006.

6. Huth JF and Eilber FR: Patterns of recurrence after resection of osteosarcoma of the extremity. Strategies for treatment of metastases. Arch Surg 124: 122-126, 1989.

7. Ward WG, Mikaelian K, Dorey F, et al: Pulmonary metastases of stage IIB extremity osteosarcoma and subsequent pulmonary metastases. J Clin Oncol 12: 1849-1858, 1994.

8. Aljubran AH, Griffin A, Pintilie $M$ and Blackstein $M$ Osteosarcoma in adolescents and adults: survival analysis with and without lung metastases. Ann Oncol 20: 1136-1141, 2009.
9. Gelderblom H, Jinks RC, Sydes M, et al; European Osteosarcoma Intergroup: Survival after recurrent osteosarcoma: data from 3 European Osteosarcoma Intergroup (EOI) randomized controlled trials. Eur J Cancer 47: 895-902, 2011.

10. Kempf-Bielack B, Bielack SS, Jürgens H, et al: Osteosarcoma relapse after combined modality therapy: an analysis of unselected patients in the Cooperative Osteosarcoma Study Group (COSS). J Clin Oncol 23: 559-568, 2005.

11. Meyers PA, Heller G, Healey JH, et al: Osteogenic sarcoma with clinically detectable metastasis at initial presentation. J Clin Oncol 11: 449-453, 1993.

12. Kager L, Zoubek A, Pötschger U, et al: Primary metastatic osteosarcoma: presentation and outcome of patients treated on neoadjuvant Cooperative Osteosarcoma Study Group protocols. J Clin Oncol 21: 2011-2018, 2003.

13. Bacci G, Briccoli A, Longhi A, et al: Treatment and outcome of recurrent osteosarcoma: experience at Rizzoli in 235 patients initially treated with neoadjuvant chemotherapy. Acta Oncol 44: 748-755, 2005

14. Mialou V, Philip T, Kalifa C, et al: Metastatic osteosarcoma at diagnosis: prognostic factors and long-term outcome - the French pediatric experience. Cancer 104: 1100-1109, 2005.

15. Ferrari S, Briccoli A, Mercuri M, et al: Postrelapse survival in osteosarcoma of the extremities: prognostic factors for long-term survival. J Clin Oncol 21: 710-715, 2003.

16. Leary SE, Wozniak AW, Billups CA, et al: Survival of pediatric patients after relapsed osteosarcoma: the St. Jude Children's Research Hospital experience. Cancer 119: 2645-2653, 2013.

17. Kimura H, Suzuki M, Ando S, et al: Pulmonary metastasectomy for osteosarcomas and soft tissue sarcomas. Cancer \& Chemotherapy 31: 1319-1323, 2004 (In Japanese).

18. Pfannschmidt J,Klode J, Muley T, Hoffmann H and Dienemann H: Pulmonary resection for metastatic osteosarcomas: a retrospective analysis of 21 patients. Thorac Cardiovasc Surg 54: 120-123, 2006.

19. Harting MT, Blakely ML, Jaffe N, et al: Long-term survival after aggressive resection of pulmonary metastases among children and adolescentce with osteosarcoma. J Pediatr Surg 41: 194-199, 2006.

20. Buddingh EP, Anninga JK, Versteegh MI, et al: Prognostic factors in pulmonary metastasized high-grade osteosarcoma. Pediatr Blood Cancer 54: 216-221, 2010.

21. Treasure T, Fiorentino F, Scarci M, Møller H and Utley M: Pulmonary metastasectomy for sarcoma: a systematic review of reported outcomes in the context of Thames Cancer Registry data. BMJ Open 2: e001736, 2012. 